\title{
THE HOMEOMORPHIC PROPERTY OF THE STOCHASTIC FLOW GENERATED BYTHE ONE-DEFAULT MODEL IN ONE DIMENSIONAL CASE
}

\section{Fatima Benziadi}

Laboratory of Stochastic Models, Statistics and Applications, University of Saida, Dr Moulay Tahar, PO.Box 138 En-Nasr, 20000 Saida, Algeria

\begin{abstract}
In this paper, we will try to study the same result proved in [10]. So, on the same model and with some assumptions, we will study the property of homeomorphism of the stochastic flow generated by the natural model in a one-dimensional case and with some modifications, based on an important theory of Hiroshi Kunita. This is the main motivation of our research.

Keywords: Credit risk, Stochastic flow, Stochastic differential geometry, Diffeomorphism.
\end{abstract}

\section{Introduction}

The notion of flow in the deterministic case for ordinary differential equations has been studied by Blagovescenski and Freindlin [11]. Under stronger assumptions of regularity of coefficients, such solutions determine a stochastic flow of diffeomorphisms. This question was discussed under variety of assumptions by Baxendal [12], Bismut [6], Elworthy [3], Kunita [2], Malliavin [4] and others. See also Kunita [13] for extensive literature on the subject. While the method of Bismut and $\mathrm{Ku}-$ nita, is primarily an extension of the original one of Blagovescenski and Freindlin on using Kolmogorov extension theorem, the original method of Elworthy [3] is based

\footnotetext{
Received April 04, 2020. accepted January 192021

Communicated by Aleksandar Nastić

Corresponding Author: Fatima Benziadi, Laboratory of Stochastic Models, Statistics and Applications, University of Saida, Dr Moulay Tahar, PO.Box 138 En-Nasr, 20000 Saida, Algeria | E-mail: fatima.benziadi@univ-saida.dz

2010 Mathematics Subject Classification. Primary 60G17; Secondary 60H05

(C) 2021 by University of Niš, Serbia | Creative Commons License: CC BY-NC-ND
} 
on using theory of stochastic integration on some appropriate Hilbert manifold of diffeomorphisms. These method originated from a similar approach in the deterministic case (but still in the framework of Hilbert manifolds) by Ebin and Marsden [14], see also Ebin [15].

The notion of the stochastic flow associated with a stochastic differential equation has been studied by several authors, e.g. Elworthy [3], Malliavin [4], IkedaWatanabe [5], Bismut [6]. In this work, we are interested in the stochastic flow generated by the so-called $\downarrow$-model, it is one-default model which gives the conditional law of a random time with respect to a reference filtration. This models are widely applied in modeling financial risk and price valuation of financial products.

Precisely, it is proved in [1] that, for any continuous local martingale $Y$, for any Lipschitz function $f$ on $\mathbb{R}$ null at the origin, there exists a probability measure $\mathbb{Q}$ and a random time $\tau>0$ on an extension of $(\Omega, \mathbb{F}, \mathbb{P})$, such that the survival probability of $\tau$, i.e., $\mathbb{Q}\left[\tau>t \mid \mathcal{F}_{t}\right]$ is equal to $Z_{t}$ for $t \geq 0$. In the last reference, it has also been shown that there exists several solutions and that an increasing family of martingales, combined with a stochastic differential equation, constitutes a natural way to construct these solutions, which means that $X_{t}^{u}=\mathbb{Q}\left[\tau \leq \mid \mathcal{F}_{t}\right], 0<u, t<\infty$ satisfies the following stochastic differential equation :

$$
\left(\natural_{u}\right):\left\{\begin{array}{l}
d X_{t}=X_{t}\left(-\frac{e^{-\Lambda_{t}}}{1-Z_{t}} d N_{t}+f\left(X_{t}-\left(1-Z_{t}\right)\right) d Y_{t}\right), \quad t \in[u, \infty) \\
X_{u}=x
\end{array}\right.
$$

where the initial condition $x$ can be any $\mathcal{F}_{u}$-mesurable random variable.

The main result of this paper is to prove the homeomorphism property of the stochastic flow generated by the stochastic flow associated with the q-equation based on Hiroshi Kunita theory, but we impose the following hypotheses:

\section{The first hypothesis:}

We keep the same naturel model, but we assume that all the processes indicated in the -equation take real values. Thus, we impose that the coefficients of this equation are Lipschitz continuous.

\section{The second hypothesis:}

We always assume the hypothesis mentioned in [1], which denoted that the stochastic integral $\int_{u}^{t} \frac{e^{-\Lambda_{s}}}{1-Z_{s}} d N_{s}, u \leq t<\infty$, exists and defines a local martingale.

Remark 1.1. With these assumptions, we recall that the solution of the -equation is continuous according to the article [7].

Remark 1.2. It is reported here that the H. Kunita theory appearing in Section 2 was done for multidimensional processes. Therefore, to obtain our result in the one-default model, it suffices to apply the unidimensional version of the Itô's formula. 


\section{The stochastic flow of stochastic differential equation}

This section is borrowed from [2].

Let $G_{1}(x), \ldots, G_{r}(x)$ be continuous mappings from $\mathbb{R}^{d}$ into itself and $M_{t}^{1}, \ldots, M_{t}^{r}$ be continuous semimartingales defined on a probability space $\left(\Omega, \mathbb{F}, \mathbb{P} ; \mathbb{F}_{t}\right)$. Here $\mathbb{F}_{t}, 0 \leq t<\infty$ is an increasing family of sub $\sigma$-fields of $\mathbb{F}$ such that $\wedge_{\varepsilon>0} \mathbb{F}_{t+\varepsilon}=\mathbb{F}_{t}$ holds for each $t$. Consider an Itô stochastic differential equation (SDE) on $\mathbb{R}^{d}$;

$$
d \xi_{t}=\sum_{j=1}^{r} G_{j}\left(\xi_{t}\right) d M_{t}^{j}
$$

A sample continuous $\mathbb{F}_{t}$-adapted stochastic process $\xi_{t}$ with values in $\mathbb{R}^{d}$ is called a solution of (2.1), if it satisfies

$$
\xi_{t}=\xi_{0}+\sum_{j=1}^{d} \int_{0}^{t} G_{j}\left(\xi_{s}\right) d M_{s}^{j}
$$

where the right hand side is the Ito integral.

Concerning coefficients of the equation, we will assume in this section that they are Lipschitz continuous, i.e., there is a positive constant $L$ such that

$$
\left|G_{j}^{i}(x)-G_{j}^{i}(y)\right| \leq L|x-y|, \quad \forall x, y \in \mathbb{R}^{d}
$$

holds for all indices $i, j$, where $G_{j}^{i}(x)$ is the $i$-th component of the vector function $G_{j}(x)$. Then for a given point $x$ of $\mathbb{R}^{d}$, the equation has a unique solution such that $\xi_{0}=0$. We denote it as $\xi_{t}(x)$ or $\xi_{t}(x, \omega)$. It is continuous in $(t, x)$ a.s. In fact, the following proposition is well known.

Proposition 2.1. [8]. $\xi_{t}(x, \omega)$ is continuous in $[0, \infty) \times \mathbb{R}^{d}$ for almost all $\omega$. Furthermore, for any $T>0$ and $p \geq 2$, there is a positive constant $K_{p, T}^{(1)}$ such that

$$
\mathbb{E}\left|\xi_{t}(x)-\xi_{s}(y)\right|^{p} \leq K_{p, T}^{(1)}\left(|x-y|^{p}+|t-s|^{\frac{p}{2}}\right)
$$

holds for all $x, y$ of $\mathbb{R}^{d}$ and $t, s$ of $[0, T]$.

We thus regard that for fixed $t, \xi_{t}(\cdot, \omega)$ is a continuous map from $\mathbb{R}^{d}$ into itself for almost all $\omega$. The purpose of this section is to prove that map $\xi_{t}(\cdot, \omega)$ is one to one and onto, and that the inverse $\operatorname{map} \xi_{t}^{-1}(\cdot, \omega)$ is also continuous.

Theorem 2.1. [2]. Suppose that $G_{1}, \ldots, G_{r}$ of equation (2.1) are Lipschitz continuous. Then the solution map $\xi_{t}(\cdot, \omega)$ is a homeomorphism of $\mathbb{R}^{d}$ for all $t$, a.s. $\omega$.

Remark 2.1. In case of one dimensional SDE, Ogura and Yamada [9] has shown the same result under a weaker condition, using a strong comparison theorem of solutions. In fact, if coefficients are Lipschitz continuous on any finite interval (local Lipschitzan) and if they are of linear growth, i.e., $\left|G_{j}(x)\right| \leq C(1+|x|)$ holds for all $x$ with some positive $C$, then the solution $\xi_{t}(\cdot, \omega)$ is homeomorphism for any $t$ a.s. 
Remark 2.2. The (local) Lipschitz continuity of coefficients is crucial for the theorem. Ogura and Yamada [9] has given an example of one dimensional SDE with $\alpha$-Hölder continuous coefficients $\left(\frac{1}{2}<\alpha<1\right)$, which has a unique strong solution but does not have the "one to one" property.

Remark 2.3. It is enough to prove the theorem in case that $M_{t}^{i}, i=1, \ldots, r$ satisfies the properties below: Let $M_{t}^{j}=B_{t}^{j}+A_{t}^{j}$ be the decomposition of semimartingale such that $B_{t}^{j}$ is a continuous local martingale and $A_{t}^{j}$ is a continuous process of bounded variation. Let $<B^{j}>_{t}$ be the quadratic variation of $B_{t}^{j}$. Then it holds for each $j$ and $\forall s<t$,

$$
A_{t}^{j}-A_{s}^{j} \leq t-s,<B^{j}>_{t}-<B^{j}>_{s} \leq t-s, \quad \forall s<t
$$

In the following discussion, condition (2.4) is always assumed. We will first show the "one to one" property. Our approach is based on several elementary inequalities.

Lemma 2.1. [2]. Let $T>0$ and $p$ be any real number. Then there is a positive constant $K_{p, T}^{(2)}$ such that $\forall x, y \in \mathbb{R}^{d}$ and $\forall t \in[0, T]$,

$$
\mathbb{E}\left|\xi_{t}(x)-\xi_{s}(y)\right|^{p} \leq K_{p, T}^{(2)}|x-y|^{p}, \quad \forall x, y \in \mathbb{R}^{d}, \quad \forall t \in[0, T]
$$

The above lemma shows that if $x \neq y$ then $\xi_{t}(x) \neq \xi_{t}(y)$ holds for all $t$ a.s. But it does not conclude that $\xi_{t}(\cdot, \omega)$ is "one to one", since the exceptional null set $N_{x, y}=\left\{\omega \mid \xi_{t}(x)=\xi_{t}(y)\right.$ for some $\left.t\right\}$ depends on the pair $(x, y)$. To overcome this point, we shall prove the following lemma.

Lemma 2.2. [8]. Set

$$
\eta(x, y)=\frac{1}{\left|\xi_{t}(x)-\xi_{t}(y)\right|}
$$

Then $\eta_{t}(x, y)$ is continuous in $[0, \infty) \times\left\{(x, y) \in \mathbb{R}^{2 d} \mid x \neq y\right\}$.

The above lemma leads immediately to the "one to one" property of the map $\xi_{t}(\cdot, \omega)$ for all $t$ a.s. We shall next consider the onto property. We first establish

Lemma 2.3. [2]. Let $T>0$ and $p$ be any real number. Then there is a positive constant $K_{p, T}^{(3)}$ such that

$$
\mathbb{E}\left(1+\left|\xi_{t}(x)\right|^{2}\right)^{p} \leq K_{p, T}^{(3)}\left(1+|x|^{2}\right)^{p}, \quad \forall x \in \mathbb{R}^{d}, \forall t \in[0, T]
$$

Remark 2.4. It holds $\left(1+|x|^{2}\right) \leq(1+|x|)^{2} \leq 2\left(1+|x|^{2}\right)$. Therefore, inequality (2.7) implies

$$
\mathbb{E}\left(1+\left|\xi_{t}(x)\right|\right)^{2 p} \leq 2^{|p|} K_{p, T}^{(3)}(1+|x|)^{2 p}
$$

Now taking negative $p$ in the above lemma, we see that $\left|\xi_{t}(x)\right|$ tends to infinity in probability as $x$ tends sequentially to infinity. We shall prove a stronger convergence. We claim 
Lemma 2.4. [2]. Let $\overline{\mathbb{R}^{d}}=\mathbb{R}^{d} \cup\{\infty\}$ be the one point compactification of $\mathbb{R}^{d}$. Set

$$
\eta_{t}(x)= \begin{cases}\frac{1}{1+\left|\xi_{t}(x)\right|} & \text { if } x \in \mathbb{R}^{d} \\ 0 & \text { if } x=\infty\end{cases}
$$

Then $\eta_{t}(x, \omega)$ is a continuous map from $[0, \infty) \times \overline{\mathbb{R}^{d}}$ into $\mathbb{R}$ a.s.

Lemma 2.5. [2]. Define a stochastic process $\bar{\xi}_{t}$ on $\overline{\mathbb{R}^{d}}=\mathbb{R}^{d} \cup\{\infty\}$ by

$$
\bar{\xi}_{t}(x)= \begin{cases}\xi_{t}(x) & \text { if } x \in \mathbb{R}^{d} \\ \infty & \text { if } x=\infty\end{cases}
$$

Then $\bar{\xi}_{t}(x)$ is continuous in $[0, \infty) \times \overline{\mathbb{R}^{d}}$.

Now the map $\bar{\xi}_{t}$ is a homeomorphism of $\overline{\mathbb{R}^{d}}$, since it is one to one, onto and continuous. Since $\infty$ is the invariant point of the map $\bar{\xi}_{t}$, we see that $\xi_{t}$ is a homeomorphism of $\mathbb{R}^{d}$. This completes the proof of Theorem 2.2 .

\section{Main result}

In our model and with the assumptions set out in Section 1, we show the homeomorphic property of the solution of the h-equation by applying the lemmas introduced by H.Kunita presented in the previous section. We take $\varepsilon=p$ and $\beta=p-n$ with $p>0$, we have for $u \leq s \leq t$ :

$$
X_{t}^{u}(x)=x+\int_{u}^{t} X_{s}\left(-\frac{e^{-\Lambda_{s}}}{1-Z_{s}}\right) d N_{s}+\int_{u}^{t} X_{s} f\left(X_{s}-\left(1-Z_{s}\right)\right) d Y_{s}
$$

We know that the quantity $f\left(X_{s}-\left(1-Z_{s}\right)\right)$ is bounded because $f$ is a Lipschitz function, but as we do not know a priori if the quantity $\left(-\frac{e^{-\Lambda_{s}}}{1-Z_{s}}\right)$ is finite or not, we introduce the stopping time $\tau_{n}=\inf \left\{t, 1-Z_{t}<\frac{1}{n}\right\}$. Therefore, we assume the process $\tilde{X}$ instead of X:

$d \tilde{X}_{t}=\tilde{X}_{t}\left(-\frac{e^{-\Lambda_{t}}}{1-Z_{t \wedge \tau_{n}}} d N_{t}+f\left(\tilde{X}_{t}-\left(1-Z_{t}\right)\right) d Y_{t}\right)$, Such as $\tilde{X}_{t}=X_{t}, \quad \forall t \leq \tau_{n}, n \in$ $\mathbb{N}$.

\subsection{Proof of the one to one property}

In this part, we will apply the lemma 2.1 to the one-default model. So if $x=y$ the inequality is clearly satisfied for any constant $\tilde{K}_{p, T}^{2}$. We shall assume $x=y$. Let $\tilde{\varepsilon}$ be an arbitrary positive number and: 


$$
\sigma_{\tilde{\varepsilon}}=\inf \left\{t>0,\left|\tilde{X}_{t}^{u}(x)-\tilde{X}_{t}^{u}(y)\right|<\tilde{\varepsilon}\right\}
$$

denote $A_{t}=\tilde{X}_{t}^{u}(x)-\tilde{X}_{t}^{u}(y)$, and we shall apply Itô's formula to the function $f(z)=|z|^{p}$. Then it holds for $t<\tilde{\varepsilon}$;

$$
\begin{aligned}
& \tilde{X}_{t}^{u}(x)=x+\int_{u}^{t} \tilde{X}_{s}\left(-\frac{e^{-\Lambda_{s}}}{1-Z_{s \wedge \tau_{n}}}\right) d N_{s}+\int_{u}^{t} \tilde{X}_{s} f\left(\tilde{X}_{s}-\left(1-Z_{s}\right)\right) d Y_{s} \\
& d \tilde{X}_{t}^{u}(x)=\tilde{X}_{t}\left(-\frac{e^{-\Lambda_{t}}}{1-Z_{t \wedge \tau_{n}}} d N_{t}+f\left(\tilde{X}_{t}-\left(1-Z_{t}\right)\right) d Y_{t}\right) \\
& \left|\tilde{X}_{t}^{u}(x)-\tilde{X}_{t}^{u}(y)\right|^{p}-|x-y|^{p}=\int_{u}^{t} \frac{\partial f}{\partial z}\left(\tilde{X}_{t}^{u}(x)-\tilde{X}_{t}^{u}(y)\right) \times \\
& \left(\tilde{X}_{s}(x)\left(-\frac{e^{-\Lambda_{s}}}{1-Z_{s \wedge \tau_{n}}}\right) d N_{s}+\tilde{X}_{s}(x) f\left(\tilde{X}_{s}(x)-\left(1-Z_{s}\right)\right) d Y_{s}-\right. \\
& \left.\tilde{X}_{s}(y)\left(-\frac{e^{-\Lambda_{s}}}{1-Z_{s \wedge \tau_{n}}}\right) d N_{s}+\tilde{X}_{s}(y) f\left(\tilde{X}_{s}(y)-\left(1-Z_{s}\right)\right) d Y_{s}\right)+ \\
& \frac{1}{2} \int_{u}^{t} \frac{\partial^{2} f}{\partial z^{2}}\left(\tilde{X}_{s}^{u}(x)-\tilde{X}_{s}^{u}(y)\right) \times \\
& {\left[\tilde{X}_{s}(x)\left(-\frac{e^{-\Lambda_{s}}}{1-Z_{s \wedge \tau_{n}}}\right) d N_{s}+\tilde{X}_{s}(x) f\left(\tilde{X}_{s}(x)-\left(1-Z_{s}\right)\right) d Y_{s}-\right.} \\
& \left.\tilde{X}_{s}(y)\left(-\frac{e^{-\Lambda_{s}}}{1-Z_{s \wedge \tau_{n}}}\right) d N_{s}+\tilde{X}_{s}(y) f\left(\tilde{X}_{s}(y)-\left(1-Z_{s}\right)\right) d Y_{s}\right]^{2} \\
& \left|\tilde{X}_{t}^{u}(x)-\tilde{X}_{t}^{u}(y)\right|^{p}-|x-y|^{p}=\int_{u}^{t} \frac{\partial f}{\partial z}\left(\tilde{X}_{t}^{u}(x)-\tilde{X}_{t}^{u}(y)\right) \times \\
& {\left[\left(\tilde{X}_{s}(x)-\tilde{X}_{s}(y)\right)\left(-\frac{e^{-\Lambda_{s}}}{1-Z_{s \wedge \tau_{n}}}\right) d N_{s}+\right.} \\
& \left.\left(\tilde{X}_{s}(x) f\left(\tilde{X}_{s}(x)-\left(1-Z_{s}\right)\right)-\tilde{X}_{s}(y) f\left(\tilde{X}_{s}(y)-\left(1-Z_{s}\right)\right)\right) d Y_{s}\right] \\
& +\frac{1}{2} \int_{u}^{t} \frac{\partial^{2} f}{\partial z^{2}}\left(\tilde{X}_{s}^{u}(x)-\tilde{X}_{s}^{u}(y)\right) \times \\
& {\left[\left(\tilde{X}_{s}(x)-\tilde{X}_{s}(y)\right)\left(-\frac{e^{-\Lambda_{s}}}{1-Z_{s \wedge \tau_{n}}}\right) d N_{s}+\right.} \\
& \left.\left(\tilde{X}_{s}(x) f\left(\tilde{X}_{s}(x)-\left(1-Z_{s}\right)\right)-\tilde{X}_{s}(y) f\left(\tilde{X}_{s}(y)-\left(1-Z_{s}\right)\right)\right) d Y_{s}\right]^{2}
\end{aligned}
$$




$$
\left|\tilde{X}_{t}^{u}(x)-\tilde{X}_{t}^{u}(y)\right|^{p}-|x-y|^{p}=\tilde{I}_{t}+\tilde{J}_{t}
$$

we start with $\tilde{I}_{t}$ :

$$
\begin{gathered}
\tilde{I}_{t}=\int_{u}^{t} \frac{\partial f}{\partial z}\left(\tilde{X}_{t}^{u}(x)-\tilde{X}_{t}^{u}(y)\right) \times \\
{\left[\left(\tilde{X}_{s}(x)-\tilde{X}_{s}(y)\right)\left(-\frac{e^{-\Lambda_{s}}}{1-Z_{s \wedge \tau_{n}}}\right) d N_{s}+\right.} \\
\left.\left(\tilde{X}_{s}(x) f\left(\tilde{X}_{s}(x)-\left(1-Z_{s}\right)\right)-\tilde{X}_{s}(y) f\left(\tilde{X}_{s}(y)-\left(1-Z_{s}\right)\right)\right) d Y_{s}\right]
\end{gathered}
$$

Noting

$$
\begin{aligned}
& \tilde{V}\left(\tilde{X}_{s}^{x}\right)=\tilde{X}_{s}(x) f\left(\tilde{X}_{s}(x)-\left(1-Z_{s}\right)\right) \\
& \tilde{V}\left(\tilde{X}_{s}^{y}\right)=\tilde{X}_{s}(y) f\left(\tilde{X}_{s}(y)-\left(1-Z_{s}\right)\right)
\end{aligned}
$$

such that

$$
\left|\tilde{V}\left(\tilde{X}_{s}^{x}\right)-\tilde{V}\left(\tilde{X}_{s}^{y}\right)\right| \leq \tilde{L}\left|\tilde{X}_{s}^{x}-\tilde{X}_{s}^{y}\right|
$$

and

$$
\frac{\partial f}{\partial z}=p|z|^{p-1}
$$

we put

$$
\tilde{I}_{t}=\tilde{I}_{t}^{1}+\tilde{I}_{t}^{2}
$$

such that

$$
\begin{gathered}
\tilde{I}_{t}^{1}=\int_{u}^{t} \frac{\partial f}{\partial z}\left(\tilde{X}_{t}^{u}(x)-\tilde{X}_{t}^{u}(y)\right)\left(\tilde{X}_{s}(x)-\tilde{X}_{s}(y)\right)\left(-\frac{e^{-\Lambda_{s}}}{1-Z_{s \wedge \tau_{n}}}\right) d N_{s} \\
\tilde{I}_{t}^{2}=\int_{u}^{t} \frac{\partial f}{\partial z}\left(\tilde{X}_{t}^{u}(x)-\tilde{X}_{t}^{u}(y)\right)\left(\tilde{V}\left(\tilde{X}_{s}^{x}\right)-\tilde{V}\left(\tilde{X}_{s}^{y}\right)\right) d Y_{s}
\end{gathered}
$$

For $\tilde{I}_{t}^{1}$, we have:

$$
\begin{aligned}
\left|\frac{\partial f}{\partial z}\left(\tilde{X}_{t}^{u}(x)-\tilde{X}_{t}^{u}(y)\right)\left(\tilde{X}_{s}(x)-\tilde{X}_{s}(y)\right)\right| & \leq|p||z|^{p-1}\left|\tilde{X}_{s}(x)-\tilde{X}_{s}(y)\right| \\
& \leq|p|\left|\tilde{X}_{s}(x)-\tilde{X}_{s}(y)\right|^{p}
\end{aligned}
$$

Therefore

$$
\tilde{I}_{t}^{1} \leq|p| \int_{u}^{t}\left|\tilde{X}_{s}(x)-\tilde{X}_{s}(y)\right|^{p} d s \times \int_{u}^{t}-\frac{e^{-\Lambda_{s}}}{1-Z_{s \wedge \tau_{n}}} d N_{s}
$$


Noting

$Q_{t}=\int_{u}^{t}-\frac{e^{-\Lambda_{s}}}{1-Z_{s \wedge \tau_{n}}} d N_{s}$, it is a local martingale according to hypothesis 1. (so called the hypothesis $\left.H_{Y}(C)[1]\right)$. So

$$
\tilde{I}_{t}^{1} \leq|p| Q_{t} \int_{u}^{t}\left|\tilde{X}_{s}(x)-\tilde{X}_{s}(y)\right|^{p} d s
$$

For $\tilde{I}_{t}^{2}$, we have:

$$
\begin{aligned}
\left|\frac{\partial f}{\partial z}\left(\tilde{X}_{t}^{u}(x)-\tilde{X}_{t}^{u}(y)\right)\left(\tilde{V}\left(\tilde{X}_{s}^{x}\right)-\tilde{V}\left(\tilde{X}_{s}^{y}\right)\right)\right| & \leq|p||z|^{p-1} \tilde{L}\left|\tilde{X}_{s}(x)-\tilde{X}_{s}(y)\right| \\
& \leq|p| \tilde{L}\left|\tilde{X}_{s}(x)-\tilde{X}_{s}(y)\right|^{p}
\end{aligned}
$$

Therefore

$$
\tilde{I}_{t}^{2} \leq|p| \tilde{L} \int_{u}^{t}\left|\tilde{X}_{s}(x)-\tilde{X}_{s}(y)\right|^{p} d s
$$

So, we have

$$
\begin{aligned}
\tilde{I}_{t}=\tilde{I}_{t}^{1}+\tilde{I}_{t}^{2} & \leq|p| Q_{t} \int_{u}^{t}\left|\tilde{X}_{s}(x)-\tilde{X}_{s}(y)\right|^{p} d s+|p| \tilde{L} \int_{u}^{t}\left|\tilde{X}_{s}(x)-\tilde{X}_{s}(y)\right|^{p} d s \\
& \leq|p| \int_{u}^{t}\left|\tilde{X}_{s}(x)-\tilde{X}_{s}(y)\right|^{p} d s\left(Q_{t}+\tilde{L}\right)
\end{aligned}
$$

Therefore, we have

$$
\left|\mathbb{E} \tilde{I}_{t \wedge \sigma_{\tilde{\varepsilon}}}\right| \leq|p|\left(Q_{t \wedge \sigma_{\tilde{\varepsilon}}}+\tilde{L}\right) \int_{u}^{t} \mathbb{E}\left|\tilde{X}_{s \wedge \sigma_{\tilde{\varepsilon}}}(x)-\tilde{X}_{s \wedge \sigma_{\tilde{\varepsilon}}}(y)\right|^{p} d s
$$

Next,

$$
\begin{gathered}
\tilde{J}_{t}=\frac{1}{2} \int_{u}^{t} \frac{\partial^{2} f}{\partial z^{2}}\left(\tilde{X}_{s}^{u}(x)-\tilde{X}_{s}^{u}(y)\right) \times \\
{\left[\left(\tilde{X}_{s}(x)-\tilde{X}_{s}(y)\right)\left(-\frac{e^{-\Lambda_{s}}}{1-Z_{s \wedge \tau_{n}}}\right) d N_{s}+\left(\tilde{V}\left(\tilde{X}_{s}^{x}\right)-\tilde{V}\left(\tilde{X}_{s}^{y}\right)\right) d Y_{s}\right]^{2}} \\
\tilde{J}_{t}=\frac{1}{2} \int_{u}^{t} \frac{\partial^{2} f}{\partial z^{2}}\left(\tilde{X}_{s}^{u}(x)-\tilde{X}_{s}^{u}(y)\right) \times \\
{\left[\left(\tilde{X}_{s}(x)-\tilde{X}_{s}(y)\right)^{2}\left(-\frac{e^{-\Lambda_{s}}}{1-Z_{s \wedge \tau_{n}}}\right)^{2} d N_{s} d N_{s}+\left(\tilde{V}\left(\tilde{X}_{s}^{x}\right)-\tilde{V}\left(\tilde{X}_{s}^{y}\right)\right)^{2} d Y_{s} d Y_{s}\right.} \\
\left.+2\left(\tilde{X}_{s}(x)-\tilde{X}_{s}(y)\right)\left(-\frac{e^{-\Lambda_{s}}}{1-Z_{s \wedge \tau_{n}}}\right)\left(\tilde{V}\left(\tilde{X}_{s}^{x}\right)-\tilde{V}\left(\tilde{X}_{s}^{y}\right)\right) d N_{s} d Y_{s}\right]
\end{gathered}
$$

Noting $\tilde{J}_{t}=\frac{1}{2}\left[\tilde{J}_{t}^{1}+\tilde{J}_{t}^{2}+\tilde{J}_{t}^{3}\right]$ such that:

$$
\tilde{J}_{t}^{1}=\int_{u}^{t} \frac{\partial^{2} f}{\partial z^{2}}\left(\tilde{X}_{s}^{u}(x)-\tilde{X}_{s}^{u}(y)\right) \times\left(\tilde{X}_{s}(x)-\tilde{X}_{s}(y)\right)^{2}\left(-\frac{e^{-\Lambda_{s}}}{1-Z_{s \wedge \tau_{n}}}\right)^{2} d N_{s} d N_{s}
$$




$$
\begin{gathered}
\tilde{J}_{t}^{2}=\int_{u}^{t} \frac{\partial^{2} f}{\partial z^{2}}\left(\tilde{X}_{s}^{u}(x)-\tilde{X}_{s}^{u}(y)\right) \times\left(\tilde{V}\left(\tilde{X}_{s}^{x}\right)-\tilde{V}\left(\tilde{X}_{s}^{y}\right)\right)^{2} d Y_{s} d Y_{s} \\
\tilde{J}_{t}^{3}=2 \int_{u}^{t} \frac{\partial^{2} f}{\partial z^{2}}\left(\tilde{X}_{s}^{u}(x)-\tilde{X}_{s}^{u}(y)\right) \times\left(\tilde{X}_{s}(x)-\tilde{X}_{s}(y)\right)\left(-\frac{e^{-\Lambda_{s}}}{1-Z_{s \wedge \tau_{n}}}\right)\left(\tilde{V}\left(\tilde{X}_{s}^{x}\right)-\tilde{V}\left(\tilde{X}_{s}^{y}\right)\right) d N_{s} d Y_{s}
\end{gathered}
$$

and note that

$$
\frac{\partial^{2} f}{\partial z^{2}}=p(p-1)|z|^{p-2}
$$

For $\tilde{J}_{t}^{1}$ we have

$$
\begin{aligned}
\left|\frac{\partial^{2} f}{\partial z^{2}}\left(\tilde{X}_{s}^{u}(x)-\tilde{X}_{s}^{u}(y)\right) \times\left(\tilde{X}_{s}(x)-\tilde{X}_{s}(y)\right)^{2}\right| & \leq\left.|p(p-1)| z\right|^{p-2} \times\left(\tilde{X}_{s}(x)-\tilde{X}_{s}(y)\right)^{2} \mid \\
& \leq|p||p-1|\left|\tilde{X}_{s}(x)-\tilde{X}_{s}(y)\right|^{p}
\end{aligned}
$$

Therefore

$$
\tilde{J}_{t}^{1} \leq|p||p-1| \int_{u}^{t}\left|\tilde{X}_{s}(x)-\tilde{X}_{s}(y)\right|^{p} d s \int_{u}^{t}\left(-\frac{e^{-\Lambda_{s}}}{1-Z_{s \wedge \tau_{n}}}\right)^{2} d N_{s} d N_{s}
$$

The hypothesis 1 . is always assumed, so

$$
\tilde{J}_{t}^{1} \leq|p||p-1| Q_{t}^{2} \int_{u}^{t}\left|\tilde{X}_{s}(x)-\tilde{X}_{s}(y)\right|^{p} d s
$$

For $\tilde{J}_{t}^{2}$ we have

$$
\begin{aligned}
\left|\frac{\partial^{2} f}{\partial z^{2}}\left(\tilde{X}_{s}^{u}(x)-\tilde{X}_{s}^{u}(y)\right) \times\left(\tilde{V}\left(\tilde{X}_{s}^{x}\right)-\tilde{V}\left(\tilde{X}_{s}^{y}\right)\right)^{2}\right| & \leq\left.|p(p-1)| z\right|^{p-2} \tilde{L}^{2}\left(\tilde{X}_{s}^{u}(x)-\tilde{X}_{s}^{u}(y)\right)^{2} \mid \\
& \leq|p||p-1| \tilde{L}^{2}\left|\tilde{X}_{s}(x)-\tilde{X}_{s}(y)\right|^{p}
\end{aligned}
$$

So

$$
\tilde{J}_{t}^{2} \leq|p||p-1| \tilde{L}^{2} \int_{u}^{t}\left|\tilde{X}_{s}(x)-\tilde{X}_{s}(y)\right|^{p} d s
$$

For $\tilde{J}_{t}^{3}$ we have

$$
\left|\frac{\partial^{2} f}{\partial z^{2}}\left(\tilde{X}_{s}^{u}(x)-\tilde{X}_{s}^{u}(y)\right) \times\left(\tilde{X}_{s}(x)-\tilde{X}_{s}(y)\right)\left(\tilde{V}\left(\tilde{X}_{s}^{x}\right)-\tilde{V}\left(\tilde{X}_{s}^{y}\right)\right)\right|
$$




$$
\begin{gathered}
\leq\left|p(p-1) z^{p-2}\left(\tilde{X}_{s}(x)-\tilde{X}_{s}(y)\right) \tilde{L}\left(\tilde{X}_{s}(x)-\tilde{X}_{s}(y)\right)\right| \\
\quad \leq\left|p(p-1) \tilde{L}\left(\tilde{X}_{s}(x)-\tilde{X}_{s}(y)\right)^{p}\right|
\end{gathered}
$$

The hypothesis 1 . is always assumed, so

$$
\tilde{J}_{t}^{3} \leq 2|p||p-1| \tilde{L} Q_{t} \int_{u}^{t}\left|\tilde{X}_{s}(x)-\tilde{X}_{s}(y)\right|^{p} d s
$$

Therefore

$$
\begin{gathered}
\tilde{J}_{t}=\frac{1}{2}\left[\tilde{J}_{t}^{1}+\tilde{J}_{t}^{2}+\tilde{J}_{t}^{3}\right] \\
\tilde{J}_{t}=\frac{1}{2}\left[|p||p-1| Q_{t}^{2} \int_{u}^{t}\left|\tilde{X}_{s}(x)-\tilde{X}_{s}(y)\right|^{p} d s+|p||p-1| \tilde{L}^{2} \int_{u}^{t}\left|\tilde{X}_{s}(x)-\tilde{X}_{s}(y)\right|^{p} d s\right. \\
\left.+2|p||p-1| \tilde{L} Q_{t} \int_{u}^{t}\left|\tilde{X}_{s}(x)-\tilde{X}_{s}(y)\right|^{p} d s\right] \\
\tilde{J}_{t} \leq \frac{1}{2}|p||p-1|\left(Q_{t}+\tilde{L}\right)^{2} \int_{u}^{t}\left|\tilde{X}_{s}(x)-\tilde{X}_{s}(y)\right|^{p} d s
\end{gathered}
$$

Therefore

$$
\left|\mathbb{E} \tilde{J}_{t \wedge \sigma \tilde{\varepsilon}}\right| \leq \frac{1}{2}|p||p-1|\left(Q_{t}+\tilde{L}\right)^{2} \int_{u}^{t} \mathbb{E}\left|\tilde{X}_{s}(x)-\tilde{X}_{s}(y)\right|^{p} d s
$$

Summing up these two inequalities 3.1 and 3.2 , we obtain

$$
\mathbb{E}\left|\tilde{X}_{t \wedge \sigma_{\tilde{\varepsilon}}}^{u}(x)-\tilde{X}_{t \wedge \sigma_{\tilde{\varepsilon}}}^{u}(y)\right|^{p} \leq|x-y|^{p}+\tilde{C}_{p} \int_{u}^{t} \mathbb{E}\left|\tilde{X}_{s}(x)-\tilde{X}_{s}(y)\right|^{p} d s
$$

where $\tilde{C}_{p}$ is a positive constant.

By Gronwall's inquality, we have:

$$
\mathbb{E}\left|\tilde{X}_{t \wedge \sigma_{\tilde{\varepsilon}}}^{u}(x)-\tilde{X}_{t \wedge \sigma_{\tilde{\varepsilon}}}^{u}(y)\right|^{p} \leq \tilde{K}_{p, u}^{(2)}|x-y|^{p}, \quad u \leq t \leq \infty
$$

such that

$$
\tilde{K}_{p, u}^{(2)}|x-y|^{p}=\exp \left(\tilde{C}_{p} u\right)
$$

Letting $\varepsilon$ tend to 0 , we have:

$$
\mathbb{E}\left|\tilde{X}_{t \wedge \sigma}^{u}(x)-\tilde{X}_{t \wedge \sigma}^{u}(y)\right|^{p} \leq \tilde{K}_{p, u}^{(2)}|x-y|^{p}
$$


where $\sigma$ is the first time such that $\tilde{X}_{t}^{u}(x)=\tilde{X}_{t}^{u}(y)$. However, it holds $\sigma=\infty$ a.s, since otherwise the left hand side would be infinity if $p<0$. The proof is complete.

The above lemma shows that if $x \neq y$ then $\tilde{X}_{t}^{u}(x) \neq \tilde{X}_{t}^{u}(y)$ holds for all $t$ a.s . But it does not conclude that $\tilde{X}_{t}(., \omega)$ is one to one, since the exceptional null set $\tilde{N}_{x, y}=\left\{\omega / \tilde{X}_{t}^{u}(x)=\tilde{X}_{t}^{u}(y)\right.$ for somet $\}$ depends on the pair $(x, y)$. To overcome this point, we shall apply the lemma 2.2 .

In this case we have:

$$
\begin{aligned}
& \tilde{X}_{t}^{u}(x)=x+\int_{u}^{t} \tilde{X}_{s}\left(-\frac{e^{-\Lambda_{s}}}{1-Z_{s \wedge \tau_{n}}}\right) d N_{s}+\int_{u}^{t} \tilde{X}_{s} f\left(\tilde{X}_{s}-\left(1-Z_{s}\right)\right) d Y_{s} \\
& \tilde{X}_{\hat{t}}^{u}(\dot{x})=\dot{x}+\int_{u}^{t} \tilde{X}_{s}\left(-\frac{e^{-\Lambda_{s}}}{1-Z_{s \wedge \tau_{n}}}\right) d N_{s}+\int_{u}^{t} \tilde{X}_{s} f\left(\tilde{X}_{s}-\left(1-Z_{s}\right)\right) d Y_{s} \\
& \tilde{X}_{t}^{u}(y)=y+\int_{u}^{t} \tilde{X}_{s}\left(-\frac{e^{-\Lambda_{s}}}{1-Z_{s \wedge \tau_{n}}}\right) d N_{s}+\int_{u}^{t} \tilde{X}_{s} f\left(\tilde{X}_{s}-\left(1-Z_{s}\right)\right) d Y_{s} \\
& \tilde{X}_{\hat{t}}^{u}(\dot{y})=\dot{y}+\int_{u}^{t} \tilde{X}_{s}\left(-\frac{e^{-\Lambda_{s}}}{1-Z_{s \wedge \tau_{n}}}\right) d N_{s}+\int_{u}^{t} \tilde{X}_{s} f\left(\tilde{X}_{s}-\left(1-Z_{s}\right)\right) d Y_{s}
\end{aligned}
$$

Putting

$$
\begin{aligned}
\tilde{\eta}_{t}(x, y) & =\frac{1}{\left|\tilde{X}_{t}^{u}(x)-\tilde{X}_{t}^{u}(y)\right|} \\
\tilde{\eta}_{t^{\prime}}\left(x^{\prime}, y^{\prime}\right) & =\frac{1}{\left|\tilde{X}_{t^{\prime}}^{u}\left(x^{\prime}\right)-\tilde{X}_{t^{\prime}}^{u}\left(y^{\prime}\right)\right|}
\end{aligned}
$$

So

$$
\begin{gathered}
\left|\tilde{\eta}_{t}(x, y)-\tilde{\eta}_{t^{\prime}}\left(x^{\prime}, y^{\prime}\right)\right|^{p}=\left|\frac{1}{\left|\tilde{X}_{t}^{u}(x)-\tilde{X}_{t}^{u}(y)\right|}-\frac{1}{\left|\tilde{X}_{t^{\prime}}^{u}\left(x^{\prime}\right)-\tilde{X}_{t^{\prime}}^{u}\left(y^{\prime}\right)\right|}\right|^{p} \\
\leq 2^{p}\left(\frac{1}{\left|\tilde{X}_{t}^{u}(x)-\tilde{X}_{t}^{u}(y)\right|}\right)^{p}\left(\frac{1}{\left|\tilde{X}_{t^{\prime}}^{u}\left(x^{\prime}\right)-\tilde{X}_{t^{\prime}}^{u}\left(y^{\prime}\right)\right|}\right)^{p}\left[\left|\tilde{X}_{t}^{u}(x)-\tilde{X}_{t^{\prime}}^{u}\left(x^{\prime}\right)\right|^{p}+\left|\tilde{X}_{t}^{u}(y)-\tilde{X}_{t^{\prime}}^{u}\left(y^{\prime}\right)\right|^{p}\right]
\end{gathered}
$$

By Hölder inequality

$$
\begin{gathered}
\mathbb{E}\left|\tilde{\eta}_{t}(x, y)-\tilde{\eta}_{t^{\prime}}\left(x^{\prime}, y^{\prime}\right)\right|^{p} \leq 2^{p}\left(\mathbb{E}\left(\tilde{\eta}_{t}(x, y)^{4 p}\right) \mathbb{E}\left(\tilde{\eta}_{t^{\prime}}\left(x^{\prime}, y^{\prime}\right)^{4 p}\right)\right)^{\frac{1}{4}} \times \\
{\left[\left(\mathbb{E}\left|\tilde{X}_{t}^{u}(x)-\tilde{X}_{t^{\prime}}^{u}\left(x^{\prime}\right)\right|^{2 p}\right)^{\frac{1}{2}}+\left(\mathbb{E}\left|\tilde{X}_{t}^{u}(y)-\tilde{X}_{t^{\prime}}^{u}\left(y^{\prime}\right)\right|^{2 p}\right)^{\frac{1}{2}}\right]}
\end{gathered}
$$

By lemme 2.1 and proposition 2.1, we have

$$
\begin{aligned}
\mathbb{E}\left|\tilde{\eta}_{t}(x, y)-\tilde{\eta}_{t^{\prime}}\left(x^{\prime}, y^{\prime}\right)\right|^{p} & \leq \tilde{C}_{p, T}|x-y|^{-p}\left|x^{\prime}-y^{\prime}\right|^{-p}\left(\left|x-x^{\prime}\right|^{p}+\left|y-y^{\prime}\right|^{p}+2\left|t-t^{\prime}\right|^{\frac{p}{2}}\right) \\
& \leq \tilde{C}_{p, T} \tilde{\delta}^{-2 p}\left(\left|x-x^{\prime}\right|^{p}+\left|y-y^{\prime}\right|^{p}+2\left|t-t^{\prime}\right|^{\frac{p}{2}}\right)
\end{aligned}
$$


if $|x-y| \geq \tilde{\delta}$ and $\left|x^{\prime}-y^{\prime}\right| \geq \tilde{\delta}$, where $\tilde{C}_{p, T}$ is a positive constant. Then by Kolmogorov theorem 2.1, $\tilde{\eta}_{t}(x, y)$ is continuous in $[0, T] \times\{(x, y) /|x-y| \geq \tilde{\delta}\}$. Since $T$ and $\tilde{\delta}$ are arbitrary positive numbers, we get the assertion. The proof is complete.

The above calculus leads immediately the one to one property of the map $\tilde{X}_{t}^{u}(., \omega)$ for all t a.s. We shall next consider the onto property.

\subsection{Proof of the onto property}

In this part we will apply the lemmas $2.3,2.4$, and 2.5 to our model.

Let $T>0$ and $p$ any real number:

$$
\begin{gathered}
\tilde{X}_{t}^{u}(x)=x+\int_{u}^{t} \tilde{X}_{s}\left(-\frac{e^{-\Lambda_{s}}}{1-Z_{s \wedge \tau_{n}}}\right) d N_{s}+\int_{u}^{t} \tilde{X}_{s} f\left(\tilde{X}_{s}-\left(1-Z_{s}\right)\right) d Y_{s} \\
d \tilde{X}_{t}^{u}(x)=\tilde{X}_{t}\left(-\frac{e^{-\Lambda_{t}}}{1-Z_{t \wedge \tau_{n}}} d N_{t}+f\left(\tilde{X}_{t}-\left(1-Z_{t}\right)\right) d Y_{t}\right)
\end{gathered}
$$

We shall apply Itô's formula to the function $f(z)=\left(1+|z|^{2}\right)^{p}$. It holds $f\left(\tilde{X}_{t}^{u}(x)\right)-f(x)=\int_{u}^{t} \frac{\partial f}{\partial z}\left(\tilde{X}_{s}^{u}(x)\right) \times$

$$
\left[\tilde{X}_{s}(x)\left(-\frac{e^{-\Lambda_{s}}}{1-Z_{s \wedge \tau_{n}}}\right) d N_{s}+\tilde{X}_{s}(x) f\left(\tilde{X}_{s}(x)-\left(1-Z_{s}\right)\right) d Y_{s}\right]+
$$

$\frac{1}{2} \int_{u}^{t} \frac{\partial^{2} f}{\partial z^{2}}\left(\tilde{X}_{s}^{u}(x)\right) \times\left[\tilde{X}_{s}(x)\left(-\frac{e^{-\Lambda_{s}}}{1-Z_{s \wedge \tau_{n}}}\right) d N_{s}+\tilde{X}_{s}(x) f\left(\tilde{X}_{s}(x)-\left(1-Z_{s}\right)\right) d Y_{s}\right]^{2}$ $f\left(\tilde{X}_{t}^{u}(x)\right)-f(x)=\tilde{I}_{t}+\tilde{J}_{t}$ such that

$$
\begin{aligned}
& \tilde{I}_{t}=\int_{u}^{t} \frac{\partial f}{\partial z}\left(\tilde{X}_{s}^{u}(x)\right) \times\left[\tilde{X}_{s}(x)\left(-\frac{e^{-\Lambda_{s}}}{1-Z_{s \wedge \tau_{n}}}\right) d N_{s}+\tilde{X}_{s}(x) f\left(\tilde{X}_{s}(x)-\left(1-Z_{s}\right)\right) d Y_{s}\right] \\
& \tilde{J}_{t}=\frac{1}{2} \int_{u}^{t} \frac{\partial^{2} f}{\partial z^{2}}\left(\tilde{X}_{s}^{u}(x)\right) \times\left[\tilde{X}_{s}(x)\left(-\frac{e^{-\Lambda_{s}}}{1-Z_{s \wedge \tau_{n}}}\right) d N_{s}+\tilde{X}_{s}(x) f\left(\tilde{X}_{s}(x)-\left(1-Z_{s}\right)\right) d Y_{s}\right]^{2}
\end{aligned}
$$

For $\tilde{I}_{t}$, we have

$$
\begin{aligned}
& \tilde{I}_{t}=\int_{u}^{t} \frac{\partial f}{\partial z}\left(\tilde{X}_{s}^{u}(x)\right) \times\left[\tilde{X}_{s}(x)\left(-\frac{e^{-\Lambda_{s}}}{1-Z_{s \wedge \tau_{n}}}\right) d N_{s}+\tilde{X}_{s}(x) f\left(\tilde{X}_{s}(x)-\left(1-Z_{s}\right)\right) d Y_{s}\right] \\
& \tilde{I}_{t}=\int_{u}^{t} \frac{\partial f}{\partial z}\left(\tilde{X}_{s}^{u}(x)\right) \tilde{X}_{s}(x)\left(-\frac{e^{-\Lambda_{s}}}{1-Z_{s \wedge \tau_{n}}}\right) d N_{s}+\int_{u}^{t} \frac{\partial f}{\partial z}\left(\tilde{X}_{s}^{u}(x)\right) \tilde{X}_{s}(x) f\left(\tilde{X}_{s}(x)-\left(1-Z_{s}\right)\right) d Y_{s} \\
& =\tilde{I}_{t}^{1}+\tilde{I}_{t}^{2} \text { such that }
\end{aligned}
$$

$$
\tilde{I}_{t}^{1}=\int_{u}^{t} \frac{\partial f}{\partial z}\left(\tilde{X}_{s}^{u}(x)\right) \tilde{X}_{s}(x)\left(-\frac{e^{-\Lambda_{s}}}{1-Z_{s \wedge \tau_{n}}}\right) d N_{s}
$$




$$
\tilde{I}_{t}^{2}=\int_{u}^{t} \frac{\partial f}{\partial z}\left(\tilde{X}_{s}^{u}(x)\right) \tilde{X}_{s}(x) f\left(\tilde{X}_{s}(x)-\left(1-Z_{s}\right)\right) d Y_{s}
$$

For $\tilde{I}_{t}^{1}$, note $\frac{\partial f}{\partial z}=2 p z\left(1+|z|^{2}\right)^{p-1}$ and the hypothesis 1 . is always assumed, so

$$
\begin{aligned}
\tilde{I}_{t}^{1}=\int_{u}^{t} \frac{\partial f}{\partial z}\left(\tilde{X}_{s}^{u}(x)\right) \tilde{X}_{s}(x)\left(-\frac{e^{-\Lambda_{s}}}{1-Z_{s \wedge \tau_{n}}}\right) d N_{s} \\
\left|\frac{\partial f}{\partial z}\left(\tilde{X}_{s}^{u}(x)\right) \tilde{X}_{s}(x)\right| \leq 2|p||z|\left(1+|z|^{2}\right)^{p-1}\left|\tilde{X}_{s}(x)\right| \\
\leq 2|p|\left(1+\left|\tilde{X}_{s}(x)\right|^{2}\right)^{p}
\end{aligned}
$$

Therefore

$$
\tilde{I}_{t}^{1} \leq 2|p| Q_{t} \int_{u}^{t}\left(1+\left|\tilde{X}_{s}(x)\right|^{2}\right)^{p} d s
$$

For $\tilde{I}_{t}^{2}$, we have

$$
\tilde{I}_{t}^{2}=\int_{u}^{t} \frac{\partial f}{\partial z}\left(\tilde{X}_{s}^{u}(x)\right) \tilde{X}_{s}(x) f\left(\tilde{X}_{s}(x)-\left(1-Z_{s}\right)\right) d Y_{s}
$$

Noting

$$
\tilde{V}\left(\tilde{X}_{s}^{x}\right)=\tilde{X}_{s}(x) f\left(\tilde{X}_{s}(x)-\left(1-Z_{s}\right)\right)
$$

Let $\tilde{K}$ be a positive constant such that

$$
\begin{aligned}
V\left(\tilde{X}_{s}^{x}\right) & \leq \tilde{K}\left(1+\left|\tilde{X}_{s}(x)\right|^{2}\right)^{\frac{1}{2}} \\
\left|\frac{\partial f}{\partial z}\left(\tilde{X}_{s}^{u}(x)\right) \tilde{X}_{s}(x) \times \tilde{V}\left(\tilde{X}_{s}^{x}\right)\right| & \leq 2|p||z|\left(1+|z|^{2}\right)^{p-1} \tilde{K}\left(1+\left|\tilde{X}_{s}(x)\right|^{2}\right)^{\frac{1}{2}} \\
& \leq 2|p| \tilde{K}\left(1+\left|\tilde{X}_{s}(x)\right|^{2}\right)^{p}
\end{aligned}
$$

So

$$
\tilde{I}_{t}^{2} \leq 2|p| \tilde{K} \int_{u}^{t}\left(1+\left|\tilde{X}_{s}(x)\right|^{2}\right)^{p} d s
$$

Therefore

$$
\begin{aligned}
\tilde{I}_{t} & \leq 2|p| Q_{t} \int_{u}^{t}\left(1+\left|\tilde{X}_{s}(x)\right|^{2}\right)^{p} d s+2|p| \tilde{K} \int_{u}^{t}\left(1+\left|\tilde{X}_{s}(x)\right|^{2}\right)^{p} d s \\
& \leq 2|p|\left(Q_{t}+\tilde{K}\right) \int_{u}^{t}\left(1+\left|\tilde{X}_{s}(x)\right|^{2}\right)^{p} d s
\end{aligned}
$$

We have

$$
\left|\mathbb{E} \tilde{I}_{t}\right| \leq 2|p|\left(Q_{t}+\tilde{K}\right) \int_{u}^{t} \mathbb{E}\left(1+\left|\tilde{X}_{s}(x)\right|^{2}\right)^{p} d s
$$


Next, for $\tilde{J}_{t}$ we have

$$
\begin{gathered}
\tilde{J}_{t}=\frac{1}{2} \int_{u}^{t} \frac{\partial^{2} f}{\partial z^{2}}\left(\tilde{X}_{s}^{u}(x)\right) \times\left[\tilde{X}_{s}(x)\left(-\frac{e^{-\Lambda_{s}}}{1-Z_{s \wedge \tau_{n}}}\right) d N_{s}+\tilde{X}_{s}(x) f\left(\tilde{X}_{s}(x)-\left(1-Z_{s}\right)\right) d Y_{s}\right]^{2} \\
\tilde{J}_{t}=\frac{1}{2} \int_{u}^{t} \frac{\partial^{2} f}{\partial z^{2}}\left(\tilde{X}_{s}^{u}(x)\right) \times\left[\tilde{X}_{s}(x)^{2}\left(-\frac{e^{-\Lambda_{s}}}{1-Z_{s \wedge \tau_{n}}}\right)^{2} d N_{s} d N_{s}+\tilde{V}\left(\tilde{X}_{s}^{x}\right)^{2} d Y_{s} d Y_{s}\right. \\
\left.+2 \tilde{X}_{s}^{u}(x)\left(-\frac{e^{-\Lambda_{s}}}{1-Z_{s \wedge \tau_{n}}}\right) \tilde{V}\left(\tilde{X}_{s}^{x}\right) d N_{s} d Y_{s}\right]
\end{gathered}
$$

Noting $\tilde{J}_{t}=\frac{1}{2}\left[\tilde{J}_{t}^{1}+\tilde{J}_{t}^{2}+\tilde{J}_{t}^{3}\right]$, such that

$$
\begin{gathered}
\tilde{J}_{t}^{1}=\int_{u}^{t} \frac{\partial^{2} f}{\partial z^{2}}\left(\tilde{X}_{s}^{u}(x)\right) \times \tilde{X}_{s}(x)^{2}\left(-\frac{e^{-\Lambda_{s}}}{1-Z_{s \wedge \tau_{n}}}\right)^{2} d N_{s} d N_{s} \\
\tilde{J}_{t}^{2}=\int_{u}^{t} \frac{\partial^{2} f}{\partial z^{2}}\left(\tilde{X}_{s}^{u}(x)\right) \times \tilde{V}\left(\tilde{X}_{s}^{x}\right)^{2} d Y_{s} d Y_{s} \\
\tilde{J}_{t}^{3}=2 \int_{u}^{t} \frac{\partial^{2} f}{\partial z^{2}}\left(\tilde{X}_{s}^{u}(x)\right) \times \tilde{X}_{s}^{u}(x)\left(-\frac{e^{-\Lambda_{s}}}{1-Z_{s \wedge \tau_{n}}}\right) \tilde{V}\left(\tilde{X}_{s}^{x}\right) d N_{s} d Y_{s}
\end{gathered}
$$

and note that

$$
\frac{\partial^{2} f}{\partial z^{2}}=2 p\left(1+|z|^{2}\right)^{p-1}+4 p(p-1) z^{2}\left(1+|z|^{2}\right)^{p-2}
$$

Then for $\tilde{J}_{t}^{1}$ we have

$$
\begin{gathered}
\tilde{J}_{t}^{1}=\int_{u}^{t} \frac{\partial^{2} f}{\partial z^{2}}\left(\tilde{X}_{s}^{u}(x)\right) \times \tilde{X}_{s}(x)^{2}\left(-\frac{e^{-\Lambda_{s}}}{1-Z_{s \wedge \tau_{n}}}\right)^{2} d N_{s} d N_{s} \\
\left|\frac{\partial^{2} f}{\partial z^{2}}\left(\tilde{X}_{s}^{u}(x)\right) \times \tilde{X}_{s}(x)^{2}\right| \leq\left|\left(2 p\left(1+|z|^{2}\right)^{p-1}+4 p(p-1) z^{2}\left(1+|z|^{2}\right)^{p-2}\right) \tilde{X}_{s}(x)^{2}\right| \\
\leq 2|p|(2(p-1)+1)\left(1+\left|\tilde{X}_{s}(x)\right|^{2}\right)^{p}
\end{gathered}
$$

Therefore

$$
\tilde{J}_{t}^{1} \leq 2|p|(2(p-1)+1) \int_{u}^{t}\left(1+\left|\tilde{X}_{s}(x)\right|^{2}\right)^{p} d s \int_{u}^{t}\left(-\frac{e^{-\Lambda_{s}}}{1-Z_{s \wedge \tau_{n}}}\right)^{2} d N_{s} d N_{s}
$$

By hypothesis 1., we have

$$
\tilde{J}_{t}^{1} \leq 2|p|(2(p-1)+1) Q_{t}^{2} \int_{u}^{t}\left(1+\left|\tilde{X}_{s}(x)\right|^{2}\right)^{p} d s
$$


For $\tilde{J}_{t}^{2}$, we have

$$
\begin{aligned}
\tilde{J}_{t}^{2}=\int_{u}^{t} & \frac{\partial^{2} f}{\partial z^{2}}\left(\tilde{X}_{s}^{u}(x)\right) \times \tilde{V}\left(\tilde{X}_{s}^{x}\right)^{2} d Y_{s} d Y_{s} \\
\left|\frac{\partial^{2} f}{\partial z^{2}}\left(\tilde{X}_{s}^{u}(x)\right) \times \tilde{V}\left(\tilde{X}_{s}^{x}\right)^{2}\right| \leq & \mid\left(2 p\left(1+|z|^{2}\right)^{p-1}+4 p(p-1) z^{2}\left(1+|z|^{2}\right)^{p-2}\right) \\
& \times \tilde{K}^{2}\left(1+\left|\tilde{X}_{s}(x)\right|^{2}\right) \mid \\
\leq & 2|p|(2(p-1)+1) \tilde{K}^{2}\left(1+\left|\tilde{X}_{s}(x)\right|^{2}\right)^{p}
\end{aligned}
$$

Therefore

$$
\tilde{J}_{t}^{2} \leq 2|p|(2(p-1)+1) \tilde{K}^{2} \int_{u}^{t}\left(1+\left|\tilde{X}_{s}(x)\right|^{2}\right)^{p} d s
$$

For $\tilde{J}_{t}^{3}$, we have

$$
\begin{aligned}
\tilde{J}_{t}^{3}=2 \int_{u}^{t} \frac{\partial^{2} f}{\partial z^{2}}\left(\tilde{X}_{s}^{u}(x)\right) \times \tilde{X}_{s}(x) & \left(-\frac{e^{-\Lambda_{s}}}{1-Z_{s \wedge \tau_{n}}}\right) \tilde{V}\left(\tilde{X}_{s}^{x}\right) d N_{s} d Y_{s} \\
\left|\frac{\partial^{2} f}{\partial z^{2}}\left(\tilde{X}_{s}^{u}(x)\right) \times \tilde{X}_{s}(x) \tilde{V}\left(\tilde{X}_{s}^{x}\right)\right| \leq & \mid\left(2 p\left(1+|z|^{2}\right)^{p-1}+4 p(p-1) z^{2}\left(1+|z|^{2}\right)^{p-2}\right) \\
& \times \tilde{K}^{2}\left(1+\left|\tilde{X}_{s}(x)\right|^{2}\right)^{\frac{1}{2}} \tilde{X}_{s}(x) \mid \\
\leq & 2|p|(2(p-1)+1) \tilde{K}\left(1+\left|\tilde{X}_{s}(x)\right|^{2}\right)^{p}
\end{aligned}
$$

The hypothesis 1 . is always assumed, so

$$
\tilde{J}_{t}^{3} \leq 4|p|(2(p-1)+1) \tilde{K} Q_{t} \int_{u}^{t}\left(1+\left|\tilde{X}_{s}(x)\right|^{2}\right)^{p} d s
$$

Therefore

$$
\begin{gathered}
\tilde{J}_{t}=\frac{1}{2}\left[\tilde{J}_{t}^{1}+\tilde{J}_{t}^{2}+\tilde{J}_{t}^{3}\right] \\
\tilde{J}_{t}=\frac{1}{2}\left[2|p|(2(p-1)+1) Q_{t}^{2} \int_{u}^{t}\left(1+\left|\tilde{X}_{s}(x)\right|^{2}\right)^{p} d s+\right. \\
2|p|(2(p-1)+1) \tilde{K}^{2} \int_{u}^{t}\left(1+\left|\tilde{X}_{s}(x)\right|^{2}\right)^{p} d s \\
\left.+4|p|(2(p-1)+1) \tilde{K} Q_{t} \int_{u}^{t}\left(1+\left|\tilde{X}_{s}(x)\right|^{2}\right)^{p} d s\right] \\
\tilde{J}_{t} \leq|p|(2(p-1)+1)\left(Q_{t}+\tilde{K}\right)^{2} \int_{u}^{t}\left(1+\left|\tilde{X}_{s}(x)\right|^{2}\right)^{p} d s
\end{gathered}
$$


So

$$
\left|\mathbb{E} \tilde{J}_{t}\right| \leq|p|(2(p-1)+1)\left(Q_{t}+\tilde{K}\right)^{2} \int_{u}^{t} \mathbb{E}\left(1+\left|\tilde{X}_{s}(x)\right|^{2}\right)^{p} d s
$$

Summing up these two inequalities 3.3 and 3.4 , we obtain

$$
\mathbb{E}\left(1+\left|\tilde{X}_{s}(x)\right|^{2}\right)^{p} \leq\left(1+|x|^{2}\right)^{p}+\text { const } \times \int_{u}^{t} \mathbb{E}\left(1+\left|\tilde{X}_{s}(x)\right|^{2}\right)^{p} d s
$$

By Gronwall's inequality, we have

$$
\mathbb{E}\left(1+\left|\tilde{X}_{s}(x)\right|^{2}\right)^{p} \leq\left(1+|x|^{2}\right)^{p} \times \exp \left(\tilde{C}_{p, u}\right)
$$

such that

$$
\tilde{C}_{p, u}=\text { const } \times \int_{u}^{t} \mathbb{E}\left(1+\left|\tilde{X}_{s}(x)\right|^{2}\right)^{p} d s
$$

and

$$
\tilde{K}_{p, u}^{3}=\exp \left(\tilde{C}_{p, u}\right)
$$

So, we have the inequality of the lemma 2.3

$$
\mathbb{E}\left(1+\left|\tilde{X}_{s}(x)\right|^{2}\right)^{p} \leq \tilde{K}_{p, u}^{3}\left(1+|x|^{2}\right)^{p}
$$

Now, taking negative $p$ in the above calculus, we see that $\left|\tilde{X}_{t}(x)\right|$ tends to infinity in probability as $x$ tends sequencially to infinity. We shall prove a stronger convergence.

Let $\overline{\mathbb{R}}=\mathbb{R} \cup\{\infty\}$ be the one point compactification of $\mathbb{R}$. Set

$$
\tilde{X}_{t}^{u}(x)=x+\int_{u}^{t} \tilde{X}_{s}\left(-\frac{e^{-\Lambda_{s}}}{1-Z_{s \wedge \tau_{n}}}\right) d N_{s}+\int_{u}^{t} \tilde{X}_{s} f\left(\tilde{X}_{s}-\left(1-Z_{s}\right)\right) d Y_{s}
$$

$\tilde{\eta}_{t}(x)= \begin{cases}\frac{1}{1+\left|\tilde{X}_{t}(x)\right|} & \text { if } x \in \mathbb{R} \\ 0 & \text { if } x=\infty\end{cases}$

Evidently $\tilde{\eta}_{t}(x)$ is continuous in $[0, \infty) \times \mathbb{R}$. Thus just to prove the continuity in the vicinity of infinity. Suppose $p>2$. It holds

$$
\left|\tilde{\eta}_{t}(x)-\tilde{\eta}_{s}(y)\right|^{p} \leq \tilde{\eta}_{t}(x)^{p} \tilde{\eta}_{s}(y)^{p}\left|\tilde{X}_{t}(x)-\tilde{X}_{s}(y)\right|^{p}
$$

By Hölder inequality, proposition 2.1 and lemma 2.3, we have

$$
\begin{aligned}
\mathbb{E}\left|\tilde{\eta}_{t}(x)-\tilde{\eta}_{s}(y)\right|^{p} & \leq\left(\mathbb{E} \tilde{\eta}_{t}(x)^{4 p}\right)^{\frac{1}{4}}\left(\mathbb{E} \tilde{\eta}_{s}(y)^{4 p}\right)^{\frac{1}{4}}\left(\mathbb{E}\left|\tilde{X}_{t}(x)-\tilde{X}_{s}(y)\right|^{2 p}\right)^{\frac{1}{2}} \\
& \leq \tilde{C}_{p, T}(1+|x|)^{-p}(1+|y|)^{-p}\left(|x-y|^{p}+|t-s|^{\frac{p}{2}}\right)
\end{aligned}
$$


if $t, s \in[0, T]$ and $x, y \in \mathbb{R}$, where $\tilde{C}_{p, T}$ is a positive constant. Set

$$
\frac{1}{x}=x^{-1}
$$

Since

We get the inequality

$$
\frac{|x-y|}{(1+|x|)(1+|y|)} \leq\left|\frac{1}{x}-\frac{1}{y}\right|
$$

$$
\mathbb{E}\left|\tilde{\eta}_{t}(x)-\tilde{\eta}_{s}(y)\right|^{p} \leq \tilde{C}_{p, T}\left(\left|\frac{1}{x}-\frac{1}{y}\right|^{p}+|t-s|^{\frac{p}{2}}\right)
$$

Define

$$
\bar{\eta}_{t}(x)=\left\{\begin{array}{cl}
\tilde{\eta}_{t}\left(\frac{1}{x}\right) & \text { if } x \neq 0 \\
0 & \text { if } x=0
\end{array}\right.
$$

Then the above inequality implies

$$
\mathbb{E}\left|\bar{\eta}_{t}(x)-\bar{\eta}_{s}(y)\right|^{p} \leq \tilde{C}_{p, T}\left(|x-y|^{p}+|t-s|^{\frac{p}{2}}\right), x \neq 0, y \neq 0
$$

In case $y=0$, we have

$$
\mathbb{E}\left|\bar{\eta}_{t}(x)\right|^{p} \leq \tilde{C}_{p, T}|x|^{p}
$$

Therefore $\bar{\eta}_{t}(x)$ is continuous in $[0, \infty) \times \mathbb{R}$ by Kolmogorov's theorem. This proves that $\tilde{\eta}_{t}(x)$ is continuous in $[0, \infty) \times$ neighborhood of infinity.

So, define a stochastic process $\bar{X}_{t}$ on $\overline{\mathbb{R}}=\mathbb{R} \cup\{\infty\}$ by

$$
\bar{X}_{t}(x)= \begin{cases}\tilde{X}_{t}(x) & \text { if } x \in \mathbb{R} \\ \infty & \text { if } x=\infty\end{cases}
$$

Then $\bar{X}_{t}(x)$ is continuous sur $[0, \infty) \times \mathbb{R}$ by the previous lemma. Thus, for each $t>0$, the map $\bar{X}_{t}(., \omega)$ is homotopic to the identity map on $\overline{\mathbb{R}}$. Then $\bar{X}_{t}(., \omega)$ is an onto map of $\overline{\mathbb{R}}$ by a well known homotopic theory. Now, the map $\bar{X}_{t}$ is a homeomorphism of $\overline{\mathbb{R}}$, since it is one to one, onto and continuous. Since $\infty$ is the invariant point of the map $\bar{X}_{t}$, we see that $\tilde{X}_{t}$ is a homeomorphism of $\mathbb{R}$. This completes the proof of theorem 2.1.

\section{R E F E R E N C E S}

1. M.JeAnblanc, S.Song: Random times with given survival probability and their $\mathbb{F}$-martingale decomposition formula. Stochastic Processes And their Applications 121 2010-2011.

2. H.Kunita: On the decomposition of solutions of stochastic differential equations. Proceeding of the LMS Symposium on Stoch.Diff.Eqs. Durham, Juillet 1980.

3. K. D. Elworthy: Stochastic dynamical systems and their flows. stochastic analysis ed. by A.Friedman and M. Pinsky, 79-95, Academic press, New York, 1978 
4. P. Malliavin: Stochastic calculus of variation and hypoelliptic operators. Kyoto, Conference, 1976, Wiley 1978, 195-263.

5. N. IKEDA-S. WATANABE: Stochastic differential equations and diffusion processes. forthcoming book.

6. J. M. Bismut: Flots stochastiques et formula de Ito-Stratonovich généralisée. C.R. Acad. Sci. Paris, t. 290, 10 mars 1980.

7. F.Benziadi, A.Kandouci: The application of Kolmogorov's theorem in the onedefault model. Journal of Mathematical Sciences and Applications E-Notes, 4 (2), 2016,71-78

8. D. W. Stroock-S. R. S. VARAdhan: Multidimensional diffusion processes, 1979, Springer-Verlag, New York.

9. Y. Ogura-T. Yamada: On the strong comparison theorem of solutions of stochastic differential equations, to appear.

10. Fatima Benziadi, Abdeldjabbar Kandouci: Homeomorphic property of the stochastic flow of a natural equation in multi-dimensional case. Communications on Stochastic Analysis, 11 (4),2017,457-478.

11. Blagovescenski Yu. N., Freindlin M.I: Some properties of diffusion processes depending on parameter, Soviet Maths., 2, 633-636 (1961).

12. Baxendale, P: Wiener processes on manifolds of maps, Poc. Royal Soc. Edinburgh, 87A, 127-152 (1980).

13. Kunita, H: Stochastic flows and stochastic differential equations, Cambridge University Press (1990).

14. Ebin, D., Marsden, J: Groups of diffeomorphisms and the motion of an incompressible fluid, Ann. of Maths. 94, no. 1, 102-163 (1970).

15. EBIn, D: The manifold of Riemannian metrics, in Preceedings of Symposium in Pure Mathematics, v. XV, Global Analysispp, pp. 11-40, AMS, Providence 1970. 